\title{
On the search for coherent radiation from radio pulsars
}

\author{
J. M. Smits ${ }^{1}$, B. W. Stappers ${ }^{2,3}$, J.-P. Macquart ${ }^{4}$, R. Ramachandran ${ }^{2,3}$, and J. Kuijpers ${ }^{1}$ \\ 1 Department of Astrophysics, University of Nijmegen, The Netherlands \\ 2 Stichting "Astron", PO Box 2, 7990 AA Dwingeloo, The Netherlands \\ 3 Astronomical Institute "Anton Pannekoek", Amsterdam, The Netherlands \\ 4 Kapteyn Institute, University of Groningen, Groningen, The Netherlands
}

Received 10 February 2003 / Accepted 6 May 2003

\begin{abstract}
We have examined data from pulsars B0950+08 and B0329+54 for evidence of temporally coherent radiation using the modified coherence function (MCF) technique of Jenet et al. (2001). We consider the influence of both instrumental bandpass and interstellar propagation effects. Even after removal of the effects due to the instrumental bandpass, we detect a signature in the MCF of our PSR B0329+54 data which is consistent with the definition of a coherent signal. However, we model the effects due to interstellar scintillation for this pulsar and show that it reproduces the observed signature. In particular, the temporal coherence time is close to the reciprocal of the decorrelation bandwidth due to diffractive scintillation. Furthermore, comparison of the coherence times of three pulsars reported by Jenet et al. (2001) with their expected diffractive decorrelation bandwidths suggests that the detection of coherence in these pulsars is also likely a result of interstellar scintillation, and is not intrinsic to the pulsars.
\end{abstract}

Key words. radiation mechanisms: general - stars: neutron - pulsars: general - pulsars: individual: B0329+54, B0950+08

\section{Introduction}

Only a few months after the discovery of the first pulsars in 1967 (Hewish et al. 1968) it was realised they were in fact neutron stars (Gold 1968; Pacini 1968). However, the emission process, responsible for the radio-frequency radiation in pulsars, remains uncertain. One characteristic of the pulsar radiation is a very high brightness temperature which can reach $10^{30} \mathrm{~K}$ (e.g. Manchester \& Taylor 1977), which implies that it is generated by a coherent mechanism. It is therefore important to identify characteristics of coherent emission in pulsar radiation. Rickett (1975) suggested that the observed radiation is well described by amplitude modulated Gaussian noise, which contains no information about the coherent nature of the radiation. Cordes (1976) later generalized this to nonGaussian shot noise. Hankins et al. (2003) have seen individual shot nano-pulses in the giant pulses from the Crab pulsar, suggesting that the intrinsic structure is less than $2 \mathrm{~ns}$.

Jenet et al. (2001) (hereafter JAP) claim to have detected the existence of coherent non-Gaussian radiation on $100 \mathrm{~ns}$ time scales for pulsars B0823+26, B0950+08 and B1133+16 in observations made at the Arecibo observatory. They used their "modified coherence function" (MCF), defined in Eq. (1) below, to find statistics inconsistent with amplitude-modulated Gaussian noise in the voltage time series from observations of these three pulsars. They show that a coherent model for pulsar radiation can account for the observed statistics. However, if the MCF of a time series of pulsar radiation shows statistics

Send offprint requests to: J. M. Smits, e-mail: roysm@astro.kun.nl of a non-Gaussian nature, it does not yet prove that the pulsar radiation itself contains non-Gaussian statistics. We have to consider the effect of the ISM on pulsar radiation and the instrumental effects on the signal after detection. JAP assert that scintillation does not influence the statistics of the signal measured by the MCF. In particular, they state that if the statistics of the intensity fluctuations are well described by Gaussian noise, the MCF ought not to exceed zero as a result of propagation effects. Scintillation, however, can give rise to a quasi-periodic fluctuation in the frequency power spectrum (see Fig. 3b). Such fluctuations in the frequency domain can be expected to influence the autocorrelations from which the MCF is constructed.

We consider here an independent data set for pulsars B0950+08 and B0329+54 to try to confirm the detection of coherence. In Sect. 2 we describe our observations and the construction of the MCF. In Sect. 3 we present an analysis of our data and investigate whether scintillation and instrumental effects do influence the MCF. Here, we also present a numerical experiment that shows that scintillation quantitatively explains the coherent features observed by JAP and compare their values of the coherence time with the inverse of the diffractive decorrelation bandwidth for each pulsar. In Sect. 4 we discuss our results and present our conclusions.

\section{Observations and analysis}

For our analysis, we use data from pulsars B0329+54 and B0950+08. These are bright pulsars and it is therefore 
Table 1. Pulsar characteristics and analysis parameters.

\begin{tabular}{lcc}
\hline \hline Pulsar & B0950+08 & B0329+54 \\
\hline Period & $0.2531 \mathrm{~s}$ & $0.7145 \mathrm{~s}$ \\
Period derivative & $8.6 \times 10^{-17}$ & $2.04959 \times 10^{-15}$ \\
Dispersion measure & $2.9702 \mathrm{pc} \mathrm{cm}^{-3}$ & $26.7765 \mathrm{pc} \mathrm{cm}^{-3}$ \\
Centre frequency & $382 \mathrm{MHz}$ & $328 \mathrm{MHz}$ \\
Bandwidth & $10 \mathrm{MHz}$ & $5 \mathrm{MHz}$ \\
Number of pulses & 2320 & 5200 \\
Size of window & $102.4 \mu \mathrm{s}$ & $204.8 \mu \mathrm{s}$ \\
Number of windows & 16 & 16 \\
Time-resolution & $50 \mathrm{~ns}$ & $100 \mathrm{~ns}$ \\
\hline
\end{tabular}

possible to detect single pulses with the high signal-to-noise ratio required to find a clear signature of coherent radiation.

These data were taken using the Westerbork Synthesis Radio Telescopes (WSRT) with its pulsar backend, PuMa (Voûte et al. 2002). In its tied array mode the WSRT is equivalent to a single dish with a diameter of $94 \mathrm{~m}$ and has a gain of $1.2 \mathrm{~K} / \mathrm{Jy}$. PSR B0950+08 was observed on 28 April 1999 at a centre frequency of $382 \mathrm{MHz}$, with a bandwidth of $10 \mathrm{MHz}$. PSR B0329+54 was observed on 13 August and 11 September 1999 at a centre frequency of $328 \mathrm{MHz}$, with a bandwidth of $5 \mathrm{MHz}$. In both observations a $10 \mathrm{MHz}$ band was Nyquist-sampled in 2 linear polarisation channels. For PSR B0329+54 the $5 \mathrm{MHz}$ band was formed by digitally filtering the data using a finite impulse response filter. After sampling, the data were 2 bit digitised. The high time resolution of $50 \mathrm{~ns}$ and $100 \mathrm{~ns}$ for pulsars B0950+08 and $\mathrm{B} 0329+54$, respectively, is necessary to study radiation which is expected to contain features with a coherence time of a few hundred nanoseconds (Jenet et al. 2001).

We remove the effects of interstellar dispersion by means of coherent dedispersion (Hankins 1971). Finally, for both pulsars, we take two sets of small successive time windows for each individual pulse. One set contains small windows centered on the peak of the average pulse profile, the other set contains a region outside the pulse, which is used for system plus sky noise corrections. The size, number of windows, and other parameters can be found in Table 1. To make sure that the periodicity of microstructure does not show up in our results as coherence, we chose a window smaller than the typical microstructure time-scale for both pulsars, which is $0.17 \mathrm{~ms}$ for PSR B0950+08 (Rickett et al. 1975) and 0.6-1.5 ms for PSR B0329+54 (Lange et al. 1998).

From these windows we calculate the MCF for real voltages. The MCF tests the fourth moment of the signal against the square of the second moment and is given by

$M_{S}(\Delta \phi) \equiv \frac{\left\langle C_{I_{S}}(\Delta \phi)\right\rangle}{\left\langle C_{I_{S}}(0)\right\rangle}-\frac{1}{3}\left(2\left[\frac{\left\langle C_{S}(\Delta \phi)\right\rangle}{\left\langle C_{S}(0)\right\rangle}\right]^{2}+1\right)$,

where $S$ is the time series of real voltages, $I_{S}=S \cdot S$ is the intensity, while $C_{S}(\Delta \phi)$ and $C_{I_{S}}(\Delta \phi)$ are the autocorrelations of $S$ and $I_{S}$, respectively. The angular brackets denote averaging over different windows and different pulses. In the analysis of JAP, $S$ is a time series of complex voltages. Although the MCF for complex voltages is different from the MCF for real voltages, physically, they are the same.

The MCF is sensitive to phase relationships between measurement points separated in time, called temporal coherence. The two polarisations are also separated and treated as different pulses. We thus obtain 32 time series from each pulse.

\subsection{Calculating the $\mathrm{MCF}$}

The MCF is sensitive to coherent features present in any time series. For pulsar radiation, such features could result when the radiation is emitted in bunches of coherently radiating particles (e.g. Ruderman \& Sutherland 1975). However, if there is extensive incoherent addition of these bunches, the resulting radiation will contain only Gaussian statistics (Cordes 1976). Because of the way the MCF is constructed (see Eq. (1)), it has a value of zero for Gaussian noise (if applied to complex data) and falls below zero with increasing delay for amplitude modulated Gaussian noise. Therefore, if the MCF of a time series becomes larger than zero, it contains statistics of a nonGaussian nature, which might give information on bunching of coherently radiating particles. To find such statistics, we wish to calculate the MCF for a noise-subtracted pulsar signal.

In order to calculate the second term of the MCF we note that the recorded on-pulse voltage time series $V(t)$ contains both the pulsar signal and system plus sky noise. It can therefore be expressed as

$V(t)=S(t)+N(t)$

where $S(t)$ is the pulsar signal and $N(t)$ is the system plus sky noise, which is contained in the off-pulse signal. Using the voltage time series of the on- and off-pulse, the average autocorrelation (AAC) of the pulsar signal and the AAC of the intensity of the pulsar signal are calculated as follows. First, one window containing one polarisation of the on-pulse of the pulsar profile is Fourier transformed to the frequency domain. The value at each frequency is multiplied by its complex conjugate and inverse Fourier transformed back into the time domain (correlation theorem). This results in the temporal autocorrelation of one window of on-pulse signal. The autocorrelations of all the windows in the total time series of on-pulse signal (containing both polarisations) are then averaged together to give the AAC of the on-pulse, $\left\langle C_{V}(\Delta \phi)\right\rangle$. The same calculation is made for the off-pulse signal to give $\left\langle C_{N}(\Delta \phi)\right\rangle$. The AAC of the pulsar signal without the system and sky noise, is given by

$\left\langle C_{S}(\Delta \phi)\right\rangle=\left\langle C_{V}(\Delta \phi)\right\rangle-\left\langle C_{N}(\Delta \phi)\right\rangle$.

This AAC is divided by the average pulsar intensity of the total time series, which is simply the average intensity of the onpulse minus the average intensity of the off-pulse,

$\left\langle I_{S}\right\rangle=\left\langle I_{V}\right\rangle-\left\langle I_{N}\right\rangle$

Squaring the result, we obtain the second term of the MCF, $\left\langle C_{S}(\Delta \phi)\right\rangle^{2} /\left\langle C_{S}(0)\right\rangle^{2}$, where $\left\langle C_{S}(0)\right\rangle=\left\langle I_{S}\right\rangle$.

To obtain the first term, we calculate the AAC's of the intensity of the voltage time series of on-pulse signal and offpulse signal. The AAC of the intensity of the noise-subtracted 


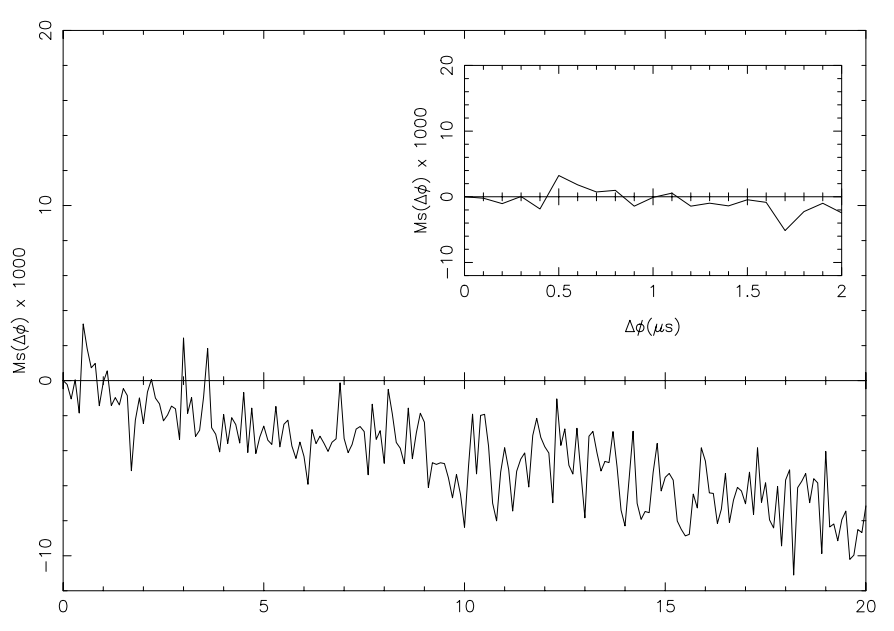

(a)

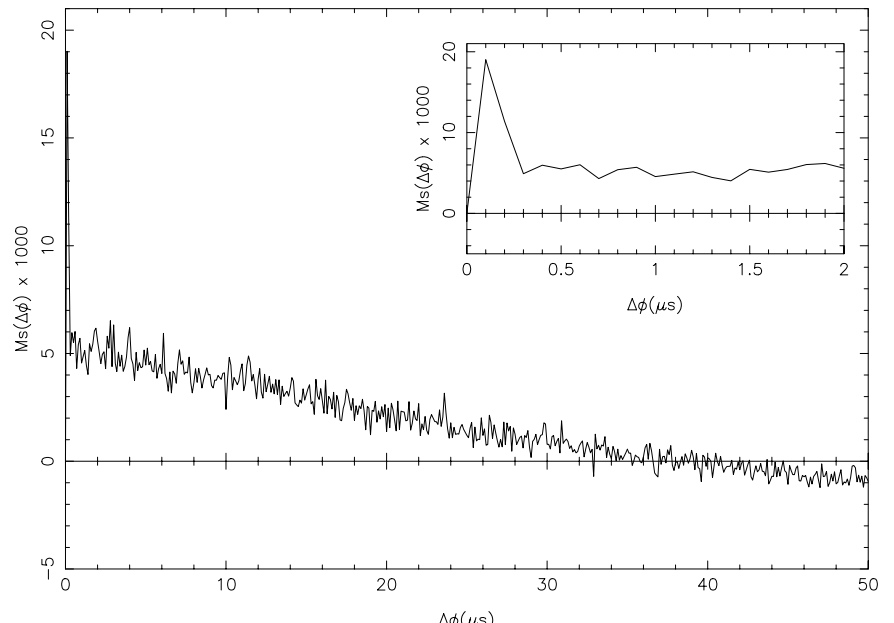

(b)

Fig. 1. Our results for the MCF as a function of phase delay calculated for pulsars B0950+08 a) and B0329+54 b) before bandpass correction, obtained from 2320 and 5200 time series of real data obtained with WSRT, respectively. The time resolution is $50 \mathrm{~ns}$ for PSR B0950+08 and $100 \mathrm{~ns}$ for PSR B0329+54. Note that the peak at zero phase delay has been set to zero.

pulsar signal is given by

$$
\begin{aligned}
\left\langle C_{I_{S}}(\Delta \phi)\right\rangle= & \left\langle C_{I_{V}}(\Delta \phi)\right\rangle-\left\langle C_{I_{N}}(\Delta \phi)\right\rangle \\
& -2\left[\left\langle C_{V}(0)\right\rangle-\left\langle C_{N}(0)\right\rangle\right] \cdot\left\langle C_{N}(0)\right\rangle \\
& -4\left[\left\langle C_{V}(\Delta \phi)\right\rangle-\left\langle C_{N}(\Delta \phi)\right\rangle\right] \cdot\left\langle C_{N}(\Delta \phi)\right\rangle .
\end{aligned}
$$

The first term in the MCF results by dividing this AAC by the average of the square of the intensity of the total time series, which is given by

$$
\begin{aligned}
\left\langle C_{I_{S}}(0)\right\rangle= & \left\langle C_{I_{V}}(0)\right\rangle-\left\langle C_{I_{N}}(0)\right\rangle \\
& -6\left[\left\langle C_{V}(0)\right\rangle-\left\langle C_{N}(0)\right\rangle\right] \cdot\left\langle C_{N}(0)\right\rangle .
\end{aligned}
$$

The MCF is then calculated according to Eq. (1).

\section{Results}

Our MCF for PSR B0950+08 is shown in Fig. 1a. The MCF starts at a value of zero and falls with increasing $\Delta \phi$, which is due to the non-zero slope of the average pulse profile of the pulsar. Our MCF for PSR B0329+54 is shown in Fig. 1b. It shows that there is a broad excess reaching a $\Delta \phi$ of $35 \mu \mathrm{s}$. There is also a large peak extending over the second and third bins.

The peak in PSR $0329+54$ is at a $\Delta \phi$ that is the reciprocal of the bandwidth. This makes us suspect that the frequencydependent gain of the system, the bandpass, can influence the MCF. We can understand the presence of a peak in the MCF due to the bandpass as follows ${ }^{1}$. Define $\tilde{S}(v)$ as the Fourier transform of the voltage signal $S(t)$ and define the bandpass as $\tilde{S}(v) \tilde{S}^{*}(v)$. The bandpass contains modulation with a width equal to the width of the bandpass, $\Delta v_{\mathrm{bp}}$ and also modulation with a width of about one third the width of the bandpass (see Figs. 3c, d). This implies that $S(t)$ and $S(t)^{2}$ contain a peak with a width on the order of $3 / \Delta v_{\mathrm{bp}}$. The autocorrelation of the signal $C_{S}(\Delta t)$ and the autocorrelation of the intensity $C_{I_{S}}(\Delta t)$ will

\footnotetext{
1 To make a clear distinction between time and frequency, we use time $(t)$ instead of phase $(\phi)$.
}

then contain a peak at $\Delta t=0$ and decorrelate on a timescale on the order of $3 / \Delta \nu_{\mathrm{bp}}$. The MCF contains the peak from the autocorrelation of the intensity minus $2 / 3$ the square of the peak of the autocorrelation of the signal itself. This last contribution works as a partial bandpass correction, but not as a full bandpass correction, as the two contributions are not necessarily equal.

\subsection{Correcting for the bandpass}

The noise power is dominated by the sky background radiation at the frequencies used in this work. We therefore take the shape of the power spectrum of the signal in the off-pulse as an estimate of the bandpass. The WSRT bandpass contains modulation with a width on the order of $2 \mathrm{MHz}$ and interference peaks (see also Kouwenhoven 2000, chapter 4). Figure 3 shows the bandpass of on-pulse and off-pulse emission for one polarisation state of pulsars B0950+08 and B0329+54, averaged over 2000 pulses.

To correct for the bandpass, we calculate and apply a bandpass correction as follows. First, we estimate the average bandpass as the power of the off-pulse signal as a function of frequency averaged over all pulses. We then normalise by dividing each frequency value by the average of the bandpass. Before the calculation of the MCF, we Fourier transform the signal inside each window into the frequency domain, divide the value at each frequency by the square root of the corresponding value of the bandpass and Fourier transform back into the time domain. This process does not correct for a possible frequency dependent phase shift introduced by the system. We carry out the procedure described above separately for both polarisations. High-level interference peaks are replaced in the frequency domain by the average of their left and right neighbours. Adjacent peaks are considered as one. Remaining interference peaks should only have a small influence on the MCF and only on long timescales. 


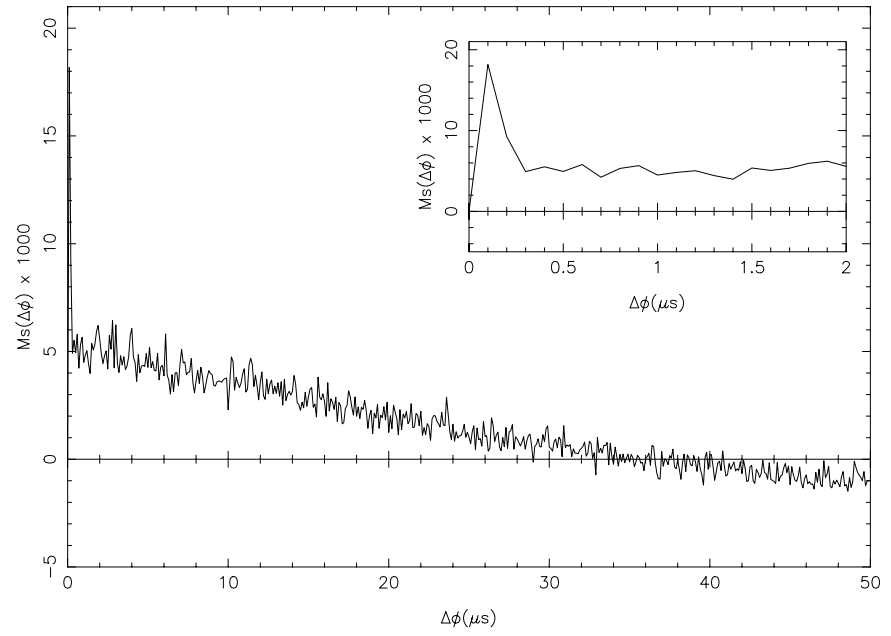

Fig. 2. Our results of the MCF calculated for PSR B0329+54 after bandpass correction. See Fig. 1 for explanation.

In Fig. 2 we see the corrected MCF for PSR B0329+54. The peak at low $\Delta \phi$ has gone down slightly, but remains present. Looking at Fig. 3b, we see that there is strong modulation in the on-pulse bandpass of PSR B0329+54 which is absent in the off-pulse bandpass. This reduces the effectiveness of the bandpass correction, as this modulation makes the onpulse bandpass different from the off-pulse bandpass. We did not do a bandpass correction for PSR B0950+08, as there is no sign of coherence present in our data from this pulsar.

\subsection{Effect of scintillation on the MCF}

Having established that variations in the bandpass shape can theoretically influence the MCF, we now consider the additional influence of interstellar scintillation (ISS). Diffractive ISS introduces structure in the instantaneous spectrum of a pulsar and can therefore, in principle, influence the MCF. In particular, we argue that ISS induces a signal in the MCF that is likely to masquerade as a false detection of temporally coherent pulsar radiation.

Diffractive interstellar scintillation is observed in pulsars, including B0950+08 and B0329+54, at centimetre wavelengths and lower. It imposes large (>100\%) temporal and spectral modulations in the intensity of the radiation. The decorrelation timescale and bandwidth of the fluctuations are chiefly determined by two parameters: the diffractive scale $r_{\text {diff }}$, and the Fresnel scale $r_{\mathrm{F}}$. It is often convenient to consider the phase fluctuations imposed by the scattering medium confined to a thin phase screen of distance $L$ from the observer. Then the diffractive scale is the distance on the phase screen over which the root mean square phase difference is one radian, and the Fresnel scale is set by the distance to the screen and the wavelength $r_{\mathrm{F}}=\sqrt{\lambda L / 2 \pi}$.

Diffractive scintillation imposes random spectral variations of characteristic bandwidth

$$
\Delta v_{\mathrm{dc}}=v\left(\frac{r_{\mathrm{diff}}}{r_{\mathrm{F}}}\right)^{2}
$$

across the pulsar signal, and this spectrum changes on a time scale $\sim r_{\text {diff }} / v$, where $v$ is the velocity of the scintillation pattern across the Earth. The expected decorrelation bandwidth for observations of B0329+54 and B0950+08 at their observing frequency are $29 \mathrm{kHz}$ (Cordes 1986) and $0.22 \mathrm{GHz}$ (Phillips \& Clegg 1992), respectively. These values were scaled from a frequency of $1 \mathrm{GHz}$ and $51 \mathrm{MHz}$, respectively, assuming $\Delta v_{\mathrm{dc}} \propto$ $v^{4.4}$ (Cordes et al. 1985).

Let us consider the effect of diffractive spectral variations on the quantity $C_{I}(\Delta t)$. The instantaneous power spectrum of the pulsar signal contains large variations with a characteristic bandwidth $\Delta v_{\mathrm{dc}}$. Thus, since the amplitudes of the Fourier transformed voltages contain ripples, the observed voltages are broadened. In the case of scintillation, the broadening can be described by the pulse broadening function (PBF) (Williamson 1972). The instantanous PBF is expected to have wiggles on a time scale set by the reciprocal of the decorrelation bandwidth, $\Delta t \approx 1 / \Delta v_{\mathrm{dc}}$. This implies that the intensity also contains variations on the same characteristic time scale. Neglecting any intrinsic temporal coherence due to the pulsar, the intensity autocorrelation function $C_{I}(\Delta t)$ is expected to peak at $\Delta t=0$ and decorrelate on a timescale set by the inverse of the scintillation decorrelation bandwidth. The average intensity autocorrelation function, obtained by combining the autocorrelations from many individual pulses and even over many diffractive timescales, is expected to exhibit the same decorrelation timescale. This is because every set of data is expected to exhibit spectral structure with a similar decorrelation bandwidth.

The second contribution to the MCF comes from the autocorrelation of the signal voltages, $C_{S}(\Delta t)$. The autocorrelation function of the pulsar voltages, $S(t)$, and the on-pulse bandpass, $\tilde{S}(v) \tilde{S}(v)^{*}$ are related by a Fourier transform:

$C_{S}(\Delta t) \rightleftharpoons\left\langle\tilde{S}(v) \tilde{S}^{*}(v)\right\rangle$,

where the angular brackets denote an average over many scintles in either/both the temporal and spectral domains. Thus the autocorrelation $C_{S}(\Delta t)$ is the inverse Fourier transform of the mean bandpass. However, the mean bandpass contains little structure due to the stochastic scintillation. Observations over many scintillation time scales wash out the frequency dependent structure due to scintillation, so that the mean on-pulse bandpass reflects mainly the instrumental response to the intrinsic spectrum of the pulsar. Thus scintillation has little effect on $C_{S}(\Delta t)$.

\subsection{The modified coherence function due to scintillation}

A set of synthetic observations from data generated by a scattering simulation is constructed in order to demonstrate the effect of scintillation on the MCF suggested by the preceeding arguments.

The simulations are conducted by constructing a set of phase fluctuations as a function of position and wavelength, $\phi(\mathbf{r}, \lambda)$, frozen onto a thin phase-changing screen located a distance $L$ from the observer's plane. We take a plane wave of unit amplitude incident on the phase screen, so that the phase 


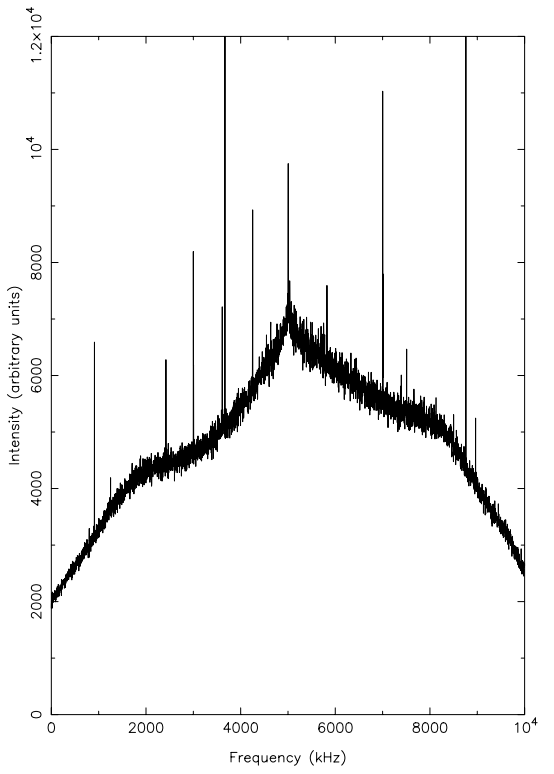

(a)

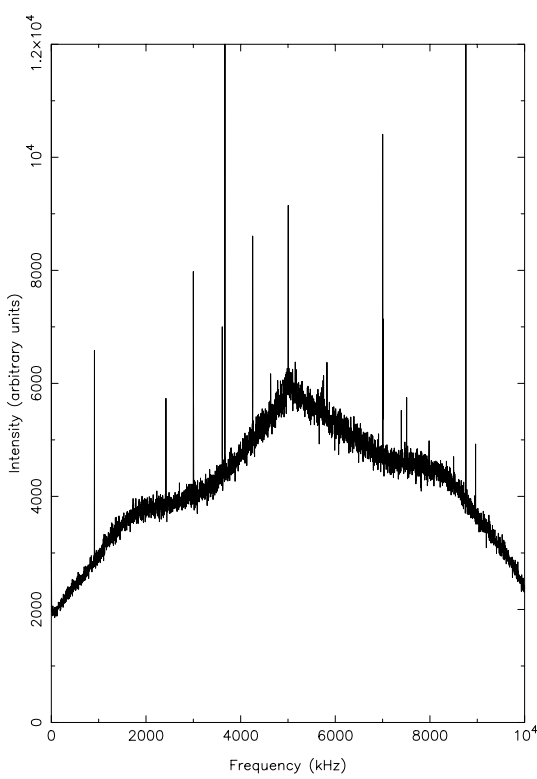

(c)

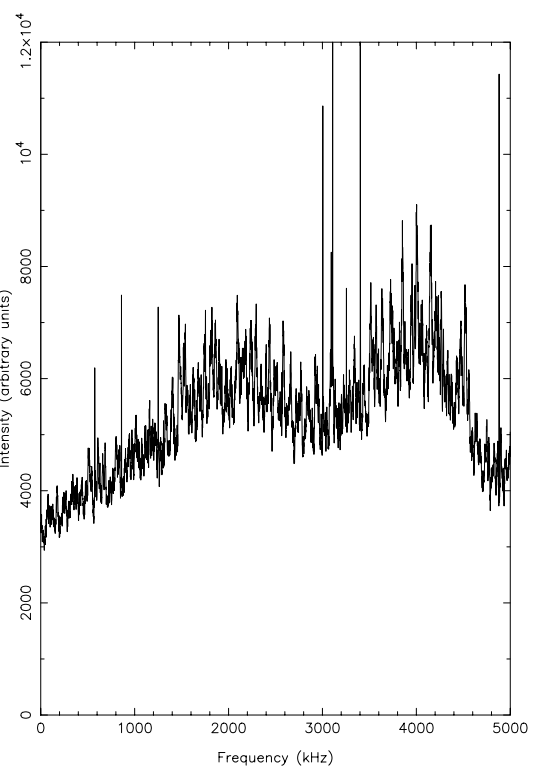

(b)

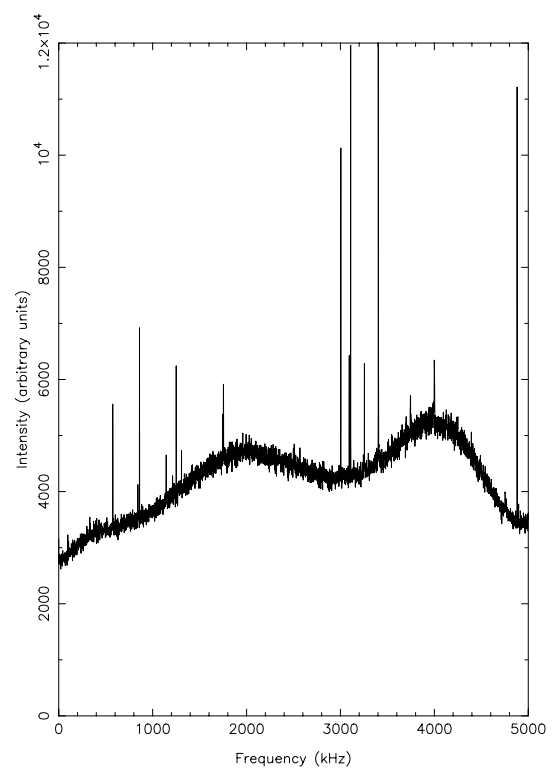

(d)

Fig. 3. Bandpass of one polarisation of WSRT for a) on-pulse of B0950+08, b) on-pulse of B0329+54, c) off-pulse of B0950+08, d) off-pulse of B0329+54, all averaged over 2000 pulses. The peaks are caused by interference. The on-pulse of B0329+54 shows scintillation with a width of approximately $30 \mathrm{kHz}$ (or $25 \mathrm{bins}$ ).

of the wave upon exiting the screen is $\phi(\boldsymbol{r})$. The phase fluctuations are generated according to a von Karman power spectrum (Goodman \& Narayan 1989)

$Q_{\phi}(\boldsymbol{q})=Q_{0}\left(|\boldsymbol{q}|^{2}+q_{\min }^{2}\right)^{-\beta / 2} \exp \left(-|\boldsymbol{q}| / q_{\max }\right)$,

where $Q_{0}$ is the amplitude of the power spectrum and sets the diffractive scale length, $r_{\text {diff }}$. The Kolmogorov value of $\beta=11 / 3$ is assumed for the power law index (Lee \& Jokipii 1975), and $q_{\max }$ and $q_{\min }$ are the upper and lower cutoffs of the power spectrum respectively. Each specific realisation of the scattering screen is generated by randomly choosing the phase associated with each spectral mode (e.g. Goodman \& Narayan 1989), so that the Fourier transform of the phase fluctuations, $\phi(x)$, is given by

$\tilde{\phi}(\boldsymbol{q})=Q_{\phi}(\boldsymbol{q})^{1 / 2} \exp [\mathrm{i}|\boldsymbol{q}| \cdot X]$,

with $X \in(0,2 \pi]$. The wavefield at the observer's plane is the inverse Fourier transform of the Fourier transform of the wavefield at the exit plane of the phase screen, $\exp [i \phi(r)]$ multiplied by the Fourier transform of the propagator $\exp \left[-\mathrm{i}\left(2 \pi r_{\mathrm{F}}\right)^{2} q^{2} / 2\right]$.

Temporal fluctuations in the wavefield are obtained by moving the phase screen relative to the observer, however, due to the limited number of grid points short time scale variations could not be simulated. Spectral variations are obtained 


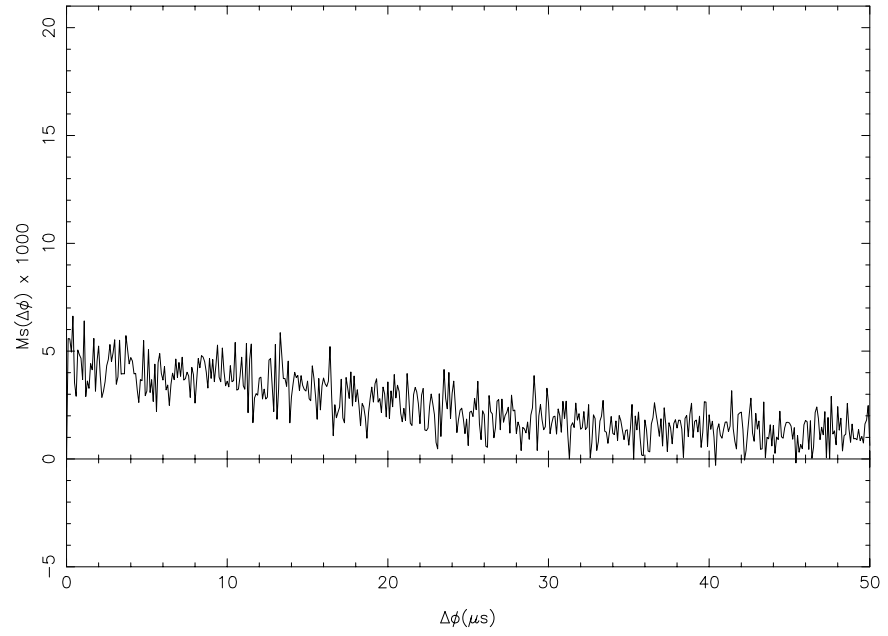

Fig. 4. The MCF of simulated scintillation at a frequency of $328 \mathrm{MHz}$ and a bandwidth of $5 \mathrm{MHz}$. The input parameters are set to generate scintillation similar to that in our data from B0329+54.

by scaling the phase fluctuations according to

$\phi(\boldsymbol{r}, \lambda)=\frac{\lambda}{\lambda_{0}} \phi\left(\boldsymbol{r}, \lambda_{0}\right)$,

and by altering the Fresnel scale in a similar manner, $r_{\mathrm{F}}(\lambda)=$ $\sqrt{\lambda / \lambda_{0}} r_{\mathrm{F}}\left(\lambda_{0}\right)$ where $\lambda$ is a wavelength within the observation bandwidth and $\lambda_{0}$ is an arbitrary, but fixed wavelength. Synthetic pulsar observations are generated by shifting the wavefield due to the scintillation pattern in time and frequency in this manner, and by choosing the length scales $r_{\mathrm{diff}}$ and $r_{\mathrm{F}}$ appropriate to the scattering conditions. The MCF is computed directly from the wavefield, where the wavenumbers, $k_{\min }$ and $k_{\max }$, were chosen to match a $5 \mathrm{MHz}$ bandwidth around $328 \mathrm{MHz}$ and the upper and lower cut-offs of the power spectrum, $q_{\min }$ and $q_{\max }$, were set at values corresponding to a physical size much larger than the scale of the phase screen and much smaller than the size of one discrete point, respectively. Table 2 shows the values of the other scintillation parameters with a brief explanation. The distance of the scintillation screen, $D$, was chosen to be in the middle between the pulsar and the observer. By tuning $Q_{0}$, we tried to create scintillation similar to that in our data from PSR B $0329+54$. The simulation results for PSR B0329+54 are shown in Fig. 4 and clearly show an excess similar to that in our MCF of PSR B0329+54. This simulation does not reproduce the peak in the first bins. However, in order to see the effects of the short time scale variations of the scintillation pattern, we have simulated a growing scintillation pattern similar to that visible in Fig. $3 \mathrm{~b}$ on top of a flat bandpass. Note that the individual modulations in the bandpass of our data from PSR B0329+54 often overlap each other. This simulation did result in a peak in the first bins of the MCF. Furthermore, we split up our data from PSR B0329+54 into smaller sets of data, calculated the MCF of each dataset and averaged them together. We did this for datasets ten to hundred times shorter than the total length of our data and saw that the peak in the first bins of the MCF decreases when the dataset becomes smaller.
Table 2. List of scintillation parameters and values used in the simulation.

\begin{tabular}{|c|c|}
\hline $\begin{array}{l}\text { Input } \\
\text { parameters }\end{array}$ & Description \\
\hline$k_{\min }=6.807 \mathrm{~m}^{-1}$ & Minimum wavenumber \\
\hline$k_{\max }=6.912 \mathrm{~m}^{-1}$ & $\begin{array}{l}\text { Maximum wavenumber } \\
\left(k_{\min } \text { and } k_{\max } \text { are the edges of }\right. \\
\text { the observed frequency range })\end{array}$ \\
\hline$D=0.7 \mathrm{kpc}$ & $\begin{array}{l}\text { Distance between scintillation } \\
\text { screen and observer }\end{array}$ \\
\hline$Q_{0}=1.1 \times 10^{-41} \mathrm{~m}^{-17 / 3}$ & $\begin{array}{l}\text { Amplitude of the power spectrum } \\
\text { of the phase fluctuations }\end{array}$ \\
\hline$N=128$ & $\begin{array}{l}\text { Number of discrete points across } \\
\text { the wavefield (total number of } \\
\text { grid points }=N^{2} \text { ) }\end{array}$ \\
\hline \multicolumn{2}{|l|}{$\begin{array}{l}\text { Measured } \\
\text { parameters }\end{array}$} \\
\hline$r_{\mathrm{F}}=1.7 \times 10^{9} \mathrm{~m}$ & Fresnel scale $(=\sqrt{D / k})$ \\
\hline$r_{\text {diff }}=3 \times 10^{7} \mathrm{~m}$ & Diffraction length scale \\
\hline$r_{\mathrm{ref}}=1.7 \times 10^{11} \mathrm{~m}$ & $\begin{array}{l}\text { Refraction length scale } \\
\left(=r_{\mathrm{F}}^{2} / r_{\mathrm{diff}}\right)\end{array}$ \\
\hline$\Delta v_{\mathrm{dc}}=0.02 \mathrm{MHz}$ & $\begin{array}{l}\text { Diffractive decorrelation } \\
\text { bandwidth }\end{array}$ \\
\hline
\end{tabular}

\section{Discussion and conclusions}

We find that frequency modulation due to scintillation and possibly the shape of the WSRT bandpass have an effect on the MCF at small delay values. This may be problematic in determining a coherence time. For PSR B0950+08 we find no signature of coherence. For PSR B0329+54 we find two features at small delay values: a peak in the first two bins and a broad excess reaching up to $35 \mu \mathrm{s}$. We have shown that the shape of the bandpass can theoretically cause a peak in the first bins of the MCF. The bandpass correction, which uses the off-pulse, might not be effective in the case of PSR B0329+54 due to the frequency modulation present in the on-pulse. By comparing Fig. 4 with Fig. 1b, we see that scintillation reproduces the broad rise observed in the MCF of PSR B0329+54. Furthermore, a simulation of an increasing scintillation pattern on top of a flat bandpass, where the modulation due to the scintillation was overlapping, has shown that small changes in the scintillation pattern can cause a peak in the first bins of the MCF. This is similar to a time dependent variation in the bandpass itself. Moreover, when smaller timescales are used to calculate the MCF the peak is seen to decrease also indicating that scintillation may play a role here.

From the above, we conclude that scintillation can be responsible for both the broad excess as well as the peak in the first two bins of the MCF of PSR B0329+54.

We now discuss whether the excess found in the MCF of three pulsars by JAP could also be due to scintillation. In Table 3 we show the similarity between the diffractive decorrelation bandwidth of the different pulsars and their 
Table 3. Diffractive decorrelation bandwidth and coherence time of pulsars B0329+54, B0823+26, B0950+08, B1133+16 and B1937+21.

\begin{tabular}{lccccc}
\hline \hline Pulsar & $\begin{array}{c}\text { Frequency } \\
(\mathrm{MHz})\end{array}$ & $\begin{array}{c}\text { Time resolution } \\
(\mathrm{ns})\end{array}$ & $\Delta v_{\mathrm{dc}}$ & $\begin{array}{c}1 / \Delta v_{\mathrm{dc}} \\
(\mu \mathrm{s})\end{array}$ & $\begin{array}{c}\text { Measured coherence } \\
\text { time }(\mu \mathrm{s})\end{array}$ \\
\hline $\mathrm{B} 0329+54$ & 328 & 100 & $29 \mathrm{kHz}[1]$ & 34 & $35[4]$ \\
$\mathrm{B} 0823+26$ & 430 & 100 & $0.81 \mathrm{MHz}[1]$ & 1.2 & $1.5[5]$ \\
$\mathrm{B} 0950+08$ & 382 & 50 & $0.22 \pm 0.03 \mathrm{GHz}[3]$ & $4.5 \times 10^{-3}$ & none [4] \\
$\mathrm{B} 0950+08$ & 430 & 100 & $0.35 \pm 0.05 \mathrm{GHz}[3]$ & $2.9 \times 10^{-3}$ & $0.4[5]$ \\
$\mathrm{B} 1133+16$ & 430 & 100 & $1.47 \mathrm{MHz}[1]$ & 0.67 & $1.1[5]$ \\
$\mathrm{B} 1937+21$ & 430 & 100 & $4.2 \pm 0.9 \mathrm{kHz}[2]$ & 238 & none [5] \\
\hline
\end{tabular}
[1] Cordes (1986).
[2] Cordes et al. (1990).
[3] Phillips \& Clegg (1992).
[4] Our data.
[5] Data from JAP.

coherence time, defined as the point where the MCF becomes zero. The values for $\Delta v_{\mathrm{dc}}$ were scaled from a frequency of $51 \mathrm{MHz}$ for PSR B0950+08 and from $1 \mathrm{GHz}$ for the other pulsars, assuming $\Delta v_{\mathrm{dc}} \propto v^{4.4}$ (Cordes et al. 1985). No errors were quoted in Cordes (1986), however it is known that $\Delta v_{\mathrm{dc}}$ can vary significantly with time, in some cases as much as a factor of 2-3 (Bhat et al. 1999). Assuming that the rise in the MCF is due to scintillation, we estimate the MCF to become zero when $\Delta \phi$ is on the order of $1 / \Delta v_{\mathrm{dc}}$. Looking at Table 3, we see that the values of the reciprocal of the diffractive decorrelation bandwidth and the measured coherence time (fourth and fifth column, respectively) are indeed similar. For our result of PSR B0950+08 there is no measured coherence time, while JAP find a coherence time for this pulsar of $0.4 \mu \mathrm{s}$. Assuming the diffractive decorrelation bandwidth from Phillips \& Clegg (1992) the time resolution of our data and that of JAP are too large to see the scintillation. The observed feature in the MCF for PSR B0950+08 of JAP (see their Fig. 1) might then result from a time variation in the bandpass of their data. There is, however, some controversy as to what the diffractive decorrelation bandwidth for PSR B0950+08 is. According to Cordes (1986) $\Delta v_{\mathrm{dc}}$ is $4.0 \mathrm{MHz}$ at an observing frequency of $430 \mathrm{MHz}$. This gives a value for $1 / \Delta v_{\mathrm{dc}}$ of $0.25 \mu \mathrm{s}$, which is in the order of size of the coherence time of $0.4 \mu \mathrm{s}$, measured by JAP. Assuming the value given by Cordes (1986), it would appear that the signal-to-noise ratio in our data is insufficient to see the scintillation, as we see no excess in the MCF. For PSR B1937+21, JAP also found no coherence time. Although this pulsar is known to scintillate, the value for $1 / \Delta v_{\mathrm{dc}}$ is so large that we can expect the excess in the MCF due to scintillation to be smeared out over such a large range of delays as to make it unmeasurable with the sensitivity of their observation.

We conclude that scintillation and possibly the shape of the bandpass causes the excess in the MCF of our data from PSR B0329+54. Furthermore, we conclude that the coherent features, found by JAP, also appear to be the result of scintillation. We therefore cannot confirm that the MCF is clearly showing us the presence of coherence in these pulsars.

Acknowledgements. The authors would like to thank J. Cordes for his extensive comments and F. A. Jenet for his helpful discussions which have both greatly contributed to the accuracy and clarity of this paper.

\section{References}

Bhat, N. D. R., Rao, A. P., \& Gupta, Y. 1999, ApJS, 121, 483

Cordes, J. M. 1976, ApJ, 210, 780

Cordes, J. M. 1986, ApJ, 311, 183

Cordes, J. M., Weisberg, J. M., \& Boriakoff, V. 1985, ApJ, 288, 221

Cordes, J. M., Wolszczan, A., Dewey, R. J., Blaskiewicz, M., \& Stinebring, D. R. 1990, ApJ, 349, 245

Gold, T. 1968, Nature, 218, 731

Goodman, J., \& Narayan, R. 1989, MNRAS, 238, 995

Hankins, T. H. 1971, ApJ, 169, 487

Hankins, T. H., Kern, J. S., Weatherall, J. C., \& Eilek, J. A. 2003, Nature, 422, 141

Hewish, A., Bell, S. J., Pilkington, J. D. H., Scott, P. F., \& Collins, R. A. 1968, Nature, 217, 709

Jenet, F. A., Anderson, S. B., \& Prince, T. A. 2001, ApJ, 558, 302

Kouwenhoven, M. L. A. 2000, Ph.D. thesis, University of Utrecht, The Netherlands

Lange, C., Kramer, M., Wielebinski, R., \& Jessner, A. 1998, A\&A, 332,111

Lee, L. C., \& Jokipii, J. R. 1975, ApJ, 201, 532

Manchester, R., \& Taylor, J. 1977

Pacini, F. 1968, Nature, 219, 145

Phillips, J. A., \& Clegg, A. W. 1992, Nature, 360, 137

Rickett, B. J. 1975, ApJ, 197, 185

Rickett, B. J., Hankins, T. H., \& Cordes, J. M. 1975, ApJ, 201, 425

Ruderman, M. A., \& Sutherland, P. G. 1975, ApJ, 196, 51

Voûte, J. L. L., Kouwenhoven, M. L. A., van Haren, P. C., et al. 2002, A\&A, 385, 733

Williamson, I. P. 1972, MNRAS, 157, 55 